\title{
FDTD Modelling of Silver Nanoparticles Embedded in Phase Separation Interface of H-PDLC
}

\author{
Kun Gui, ${ }^{1,2}$ Jihong Zheng, ${ }^{1,2}$ Kangni Wang, ${ }^{1,2}$ Daoping Li, ${ }^{1,2}$ and Songlin Zhuang ${ }^{1,2}$ \\ ${ }^{1}$ Engineering Research Center of Optical Instrument and System, Ministry of Education, Shanghai Key Lab of Modern Optical System, \\ Shanghai 200093, China \\ ${ }^{2}$ University of Shanghai for Science and Technology, Shanghai 200093, China
}

Correspondence should be addressed to Jihong Zheng; jihongzheng@sina.com

Received 28 November 2014; Revised 9 February 2015; Accepted 13 February 2015

Academic Editor: Yanjun Liu

Copyright (C) 2015 Kun Gui et al. This is an open access article distributed under the Creative Commons Attribution License, which permits unrestricted use, distribution, and reproduction in any medium, provided the original work is properly cited.

\begin{abstract}
We report localized surface plasmon resonance (LSPR) of silver nanoparticles (NPs) embedded in interface of phase separation of holographic polymer-dispersed liquid crystal (H-PDLC) gratings using Finite-Difference Time Domain method. We show that silver NPs exhibit double resonance peak at the interface, and these peaks are influenced by the angle of incident light. We observe a blue shift of the wavelength of resonance peak as the incident angle increases. However, the location of silver NPs at the interface has nearly no effect on the wavelength of resonance peak. Also we show near-field and far-field properties surrounding silver NPs and find that field distribution can be controlled through rotation of incident angle. Therefore, LSPR properties of silver NPs within H-PDLC gratings can be excited by appropriate wavelength and angle of the incident light.
\end{abstract}

\section{Introduction}

A large number of free electrons at the interior and surface of metal form free electron group, namely, plasma, and surface plasma refers in particular to the electron group existing at the surface. When the light is incident on metallic nanostructure, resonance between incident light and vibration of electron group produces surface plasmon resonance (SPR), which is reflected by a strong absorption or scattering peak on spectrum macroscopically. Localized surface plasmon resonance (LSPR) describes the properties of metallic nanoparticles (NPs) such as gold and silver nanoparticles (NPs) and is closely influenced by physicochemical properties of surrounding media. Structures and devices based on LSPR properties are attracting considerable attention as a result of their unique manipulation and control of photon at nanoscale size. It provides an effective road for nanophotonics devices and all-optical integration [1,2]. In addition, it is well-known that resonance peak of LSPR can be controlled through geometrical size of metallic NPs and surrounding media [3$6]$.
As a new type of electrooptical material, holographic polymer-dispersed liquid crystal (H-PDLC) has many potential applications in optical communications, flat panel displays, information storage, and integrated optics [7-13]. A number of practical and effective instruments have been fabricated based on the unique characteristics of H-PDLCs such as optical attenuator, holographic zoom lens, and holographic volume gratings [14-20]. Two coherent recording beams create a sinusoidal interference optical pattern, which leads to periodic phase separation between prepolymer monomers and liquid crystal (LC). Periodic alternating LC-rich region and polymer-rich region, corresponding to the interference optical pattern, form in H-PDLC gratings.

Recently, some dopants like conductive polymer [21], element fluorine [22], and surfactant molecules [23, 24] have been added to pure PDLC to improve the characteristics of the H-PDLC. Metallic NPs like silver NPs are also among the dopants. However, NPs have been only added to research the conductivity and the dielectric of the mixture [25]. Due to the LSPR properties, surface enhancement field of silver NPs has potential effect on the characteristic of the grating. 


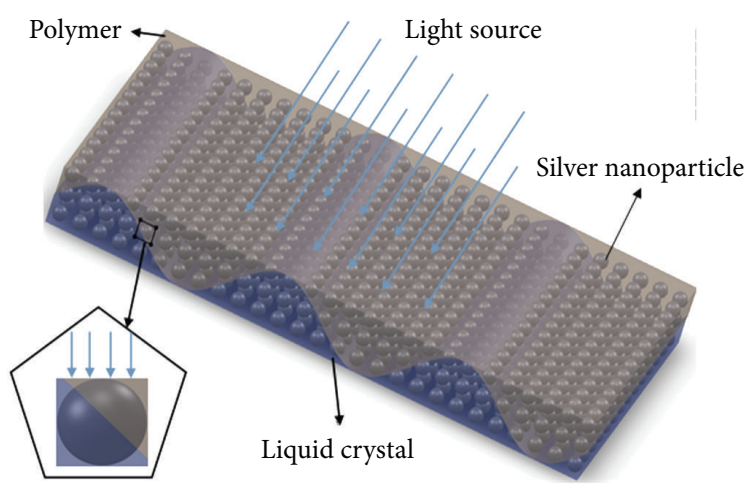

FIGURE 1: Simulation structure of H-PDLC grating doped with silver nanoparticles.

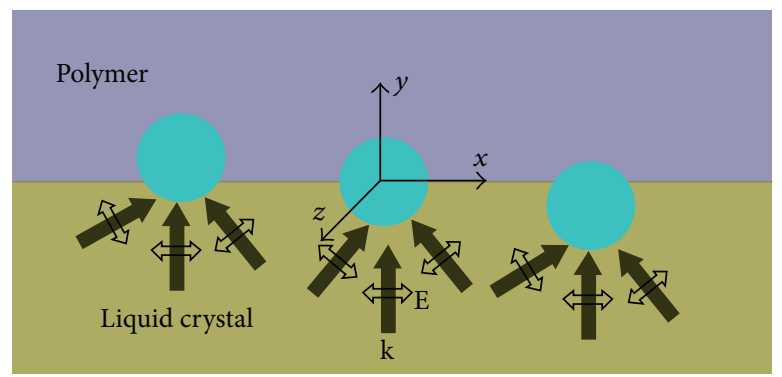

FIGURE 2: Nanoparticles in different positions and different angles of incident light.

In H-PDLC gratings doped with silver NPs, nanoparticles often disperse in polymer, LC, and the interface of polymer and LC. LSPR of silver NPs embedded in uniform medium like polymer or LC has also been researched [26]. However, we investigated LSPR of silver NPs embedded in the interface of phase separation of H-PDLC gratings using Finite-Difference Time Domain (FDTD) method. We simulated extinction, absorption, and scattering spectrum of silver NPs in this structure. We also studied the variation of the wavelength peak with the increase of incident angle and the position of silver NPs. Field distribution including near-field and far-field of silver NPs at the interface was also investigated.

\section{Materials and Methods}

2.1. Structure of H-PDLC Grating and Model of LSPR. The simulation structure is the silver NPs with diameter of $50 \mathrm{~nm}$ uniformly embedded in the mixture consisting of acrylic monomer (EB8301, $\left.n_{p}=1.5\right)$ and nematic LC (99.9\% TEB50 $+0.1 \%$ CB15 mixed liquid crystal, $n_{e}=1.7, n_{o}=1.5$ ) that is sandwiched between two pieces of glass substrate. Then the mixture is exposed in the coherent optical path to form H-PDLC gratings doped with small concentration of silver NPs. The structure is shown in Figure 1: silver NPs uniformly embedded in the H-PDLC grating excite LSPR and a monochromatic plane wave is vertically incident on the grating. Even though silver NPs are perhaps dispersed in LC, polymer, and the interface of them, our simulation is especially for the silver NPs trapped in the phase separation interface.
The H-PDLC grating consists of alternative layer of polymer and LC. Even though the phase separation interface is sinusoidal, the relative small size of silver NPs to the PDLC cell and the phase separation thickness lead to an equivalent plane. Therefore, we used a plane to illustrate the interface between polymer and LC. The equivalent plane on a small scale is shown in the inset of Figure 1. Different position of NPs trapped in the interface of the grating means different incident angle irradiating on the silver NPs, as shown in Figure 2, and the wavelength of resonance peak and field distribution will also change.

2.2. FDTD Method. The FDTD approach is a common computational method. Discretization of Maxwell equations can be adopted to solve the field distribution in different locations and at different points of time by the FDTD method in both time domain and space domain. In this paper, FDTD method is used to solve the scattering, absorption, and extinction spectrum of LSPR of silver NPs at the interface. All calculations are simulated by the FDTD solution software.

NPs embedded in the interface of polymer and LC are shown in Figure 1. With different location of silver NPs at the interface of the grating, the NPs are irradiated by a plane wave at different incident angles. The vibration direction of light waves is always in the $X-Y$ plane. The electric field intensity and extinction spectrum enhanced by silver NPs are solved by this model. In order to reduce simulation time of FDTD software, 2D-FDTD method is used. The simulation process uses perfectly matched boundary conditions and the simulation time is set to $200 \mathrm{fs}$ that is enough to make 


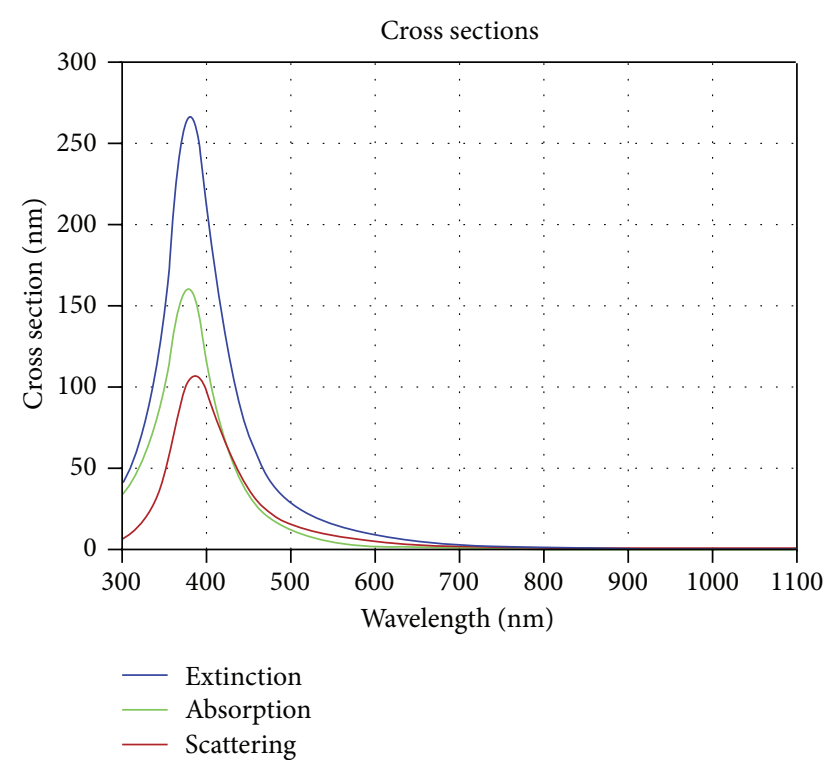

(a)

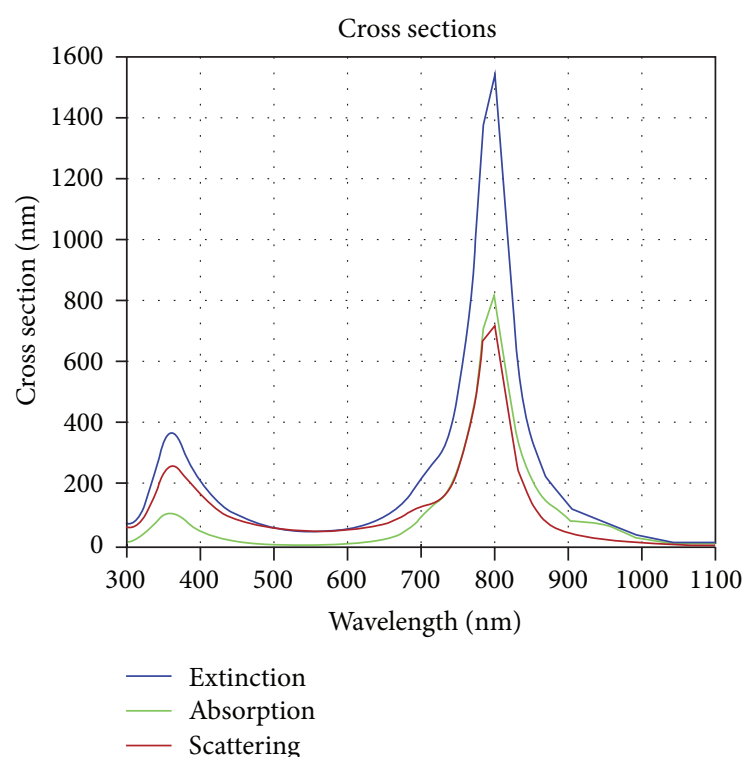

(b)

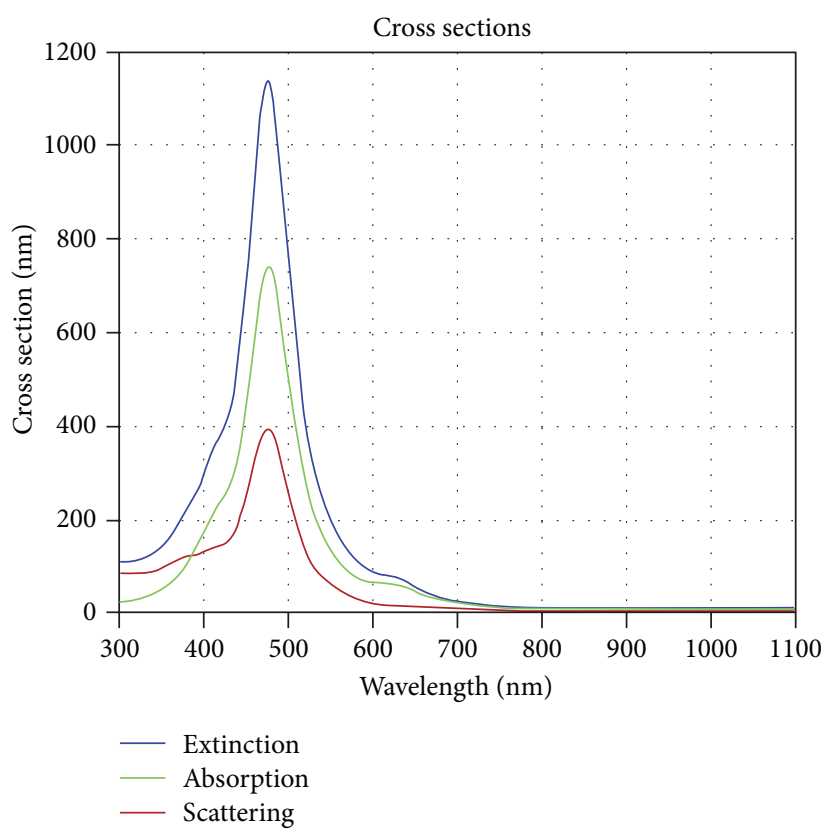

(c)

FIGURE 3: Simulated LSPR extinction spectrum of different interface: (a) homogeneous isotropic media $(n=1.5)$, (b) interface of isotropic media $\left(n_{1}=1.5, n_{2}=5\right)$, and (c) interface of isotropic medium $(n=1.5)$ and anisotropic medium $\left(n_{e}=5, n_{o}=1.5\right)$.

the eliminated evanescent field. The mesh size is $1 \mathrm{~nm} \times 1 \mathrm{~nm}$. The silver NPs called Ag-CRC from the material library of the software are used and their diameter is $50 \mathrm{~nm}$. The ordinary and extraordinary refractive indexes of LC are, respectively, 1.5 and 1.7 , and the polymer refractive index is 1.5.

\section{Results and Discussion}

When the light is incident on the H-PDLC gratings doped with silver NPs, the metal NPs will excite coherent oscillations of free electrons, resulting in the LSPR effect. When the NPs are trapped in the interface of two different materials, two distinct resonance peaks appear [27]. To discuss the LSPR of NPs in the interface of different medium, we select three kinds of distinct interface. Because the resonance characteristic of the LSPR in phase separation interface is not obvious for small refractive index difference of LC, we select uniaxial crystal materials $\left(n_{e}=5, n_{o}=1.5\right)$ instead of the ordinary LC to study the resonance characteristic. The three kinds of interface are listed as follows: interface of uniform isotropic media $(n=1.5)$, interface of isotropic media $\left(n_{1}=1.5\right.$, $\left.n_{2}=5\right)$, and interface of isotropic media $(n=1.5)$ and 


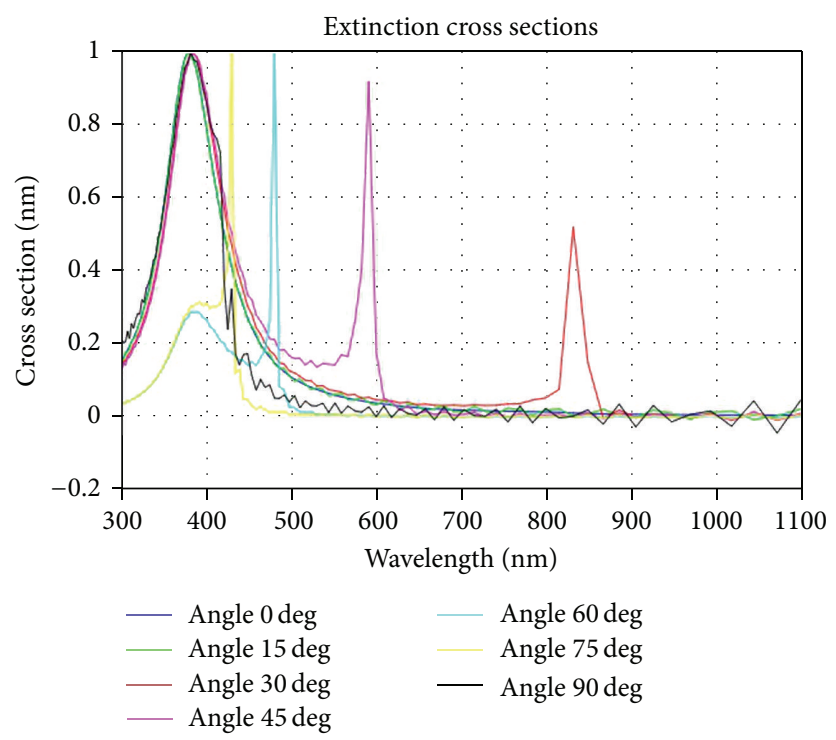

FIGURE 4: Extinction spectrum of silver nanoparticles with different incident angles (far-field characteristics).

TABLE 1: Resonance peak wavelength of different angles.

\begin{tabular}{lccccccc}
\hline Angle (degree) & 0 & 15 & 30 & 45 & 60 & 75 & 90 \\
\hline Resonance wavelength 1 (nm) & 380 & 380 & 380 & 380 & 380 & 380 & 380 \\
Resonance wavelength 2 (nm) & $/$ & $/$ & 832 & 591 & 479 & 429 & 416 \\
\hline
\end{tabular}

anisotropic media $\left(n_{e}=5, n_{o}=1.5\right)$. The simulated LSPR extinction spectrum of three kinds of interface is shown in Figure 3.

According to Figure 3, the position of resonance peak changes in the interface of anisotropic dielectric and isotropic dielectric media compared to the interface of isotropic dielectric. One peak is almost unchanged, and the other peak appears as a blue shift. Because special anisotropic dielectric media like LC have two directions of anisotropy, different dipole polarization of two directions results in the shift of resonance peak. The effective refractive index of anisotropic dielectric like LC is between ordinary and extraordinary refractive indexes. Therefore there will be a blue shift of the peak in the interface of polymer and LC.

LSPR peak of silver NPs embedded in the interface of polymer and LC will change with the angle of incident light. To reflect the variation of the resonance peak position clearly, the data were normalized as shown in Figure 4. When the angle of incident light is 0 degrees, there is only one resonance peak. Due to small anisotropy of LC, silver NPs in the interface of two media excite two close resonance peaks that seem to be a single peak. With the increase of angle, extinction spectrum appears a long wavelength peak and there is gradually a blue shift at the long Wavelength peak. Wavelength of resonance peak at different angles is shown in Table 1. Resonance wavelength 1 is the short wavelength of the resonance peak and resonance wavelength 2 is the long wavelength of the resonance peak.

To discuss the blue shift clearly, near-field distribution of silver NPs within this structure is shown in Figure 5. With the variation of incident angle, the near-field distribution at the position of unchanged resonance peak is along the direction of incident light. However, the near-field distribution at the position of blue-shift resonance peak is along the direction of the horizontal interface. In detail, the electric field component of incident light along the direction of the horizontal interface has a huge effect on the position of resonance peak.

In addition, the silver NPs may exist in different position of the interface of LC and polymer. Different locations may affect the resonance peak. Through the simulation, far-field extinction spectrum is shown in Figure 6. The position of the resonance peak has no shift when silver NPs are at different position, which is mainly due to the small refractive index difference between the polymer and LC. Thus the position of NPs at the interface almost has no effect on the wavelength of resonance peak.

\section{Conclusions}

This paper studies the LSPR effect of silver nanoparticles embedded in the phase separation interface of polymer and LC in the H-PDLC grating. With the change of the incident light angle and the position of nanoparticles at the interface, the resonance effect will be changed. With the increase of incident angle, the resonance effect appears as a long wavelength peak and achieves gradual blue shift. The resonance of the nanoparticles is hardly affected by the position of the nanoparticles at the interface. The LSPR effect of NPs in the interface of anisotropic and isotropic media 


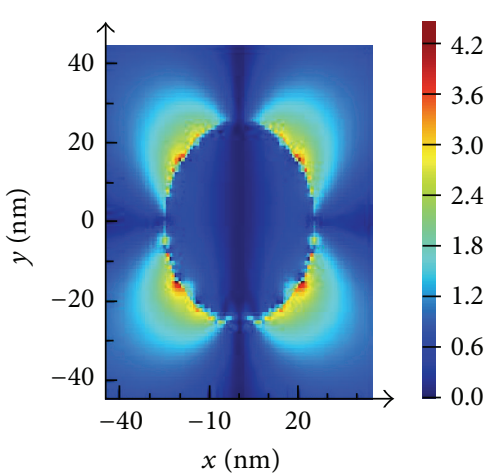

(a) 0 degrees

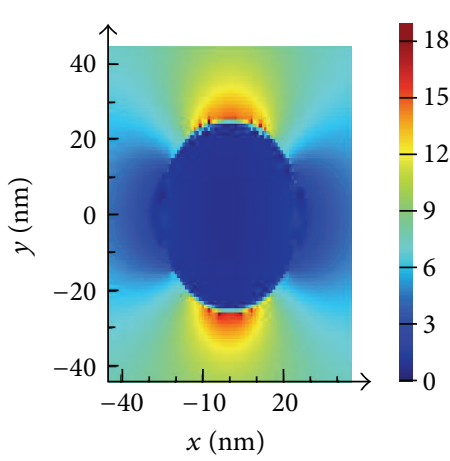

(d) 30 degrees- $832 \mathrm{~nm}$

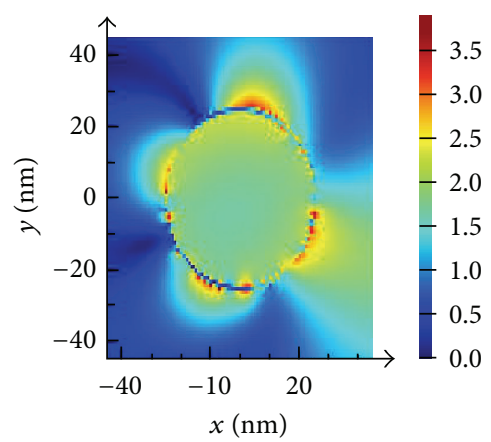

(g) 60 degrees $-380 \mathrm{~nm}$

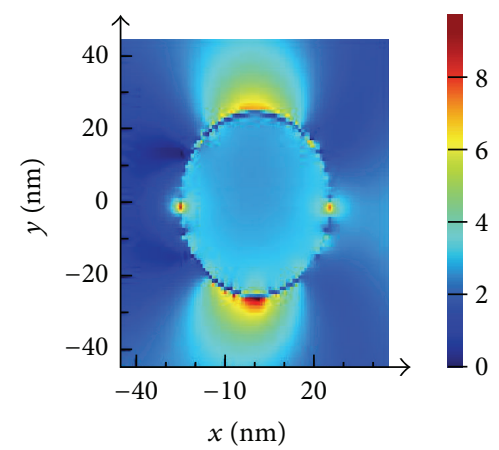

(j) 75 degrees $-429 \mathrm{~nm}$

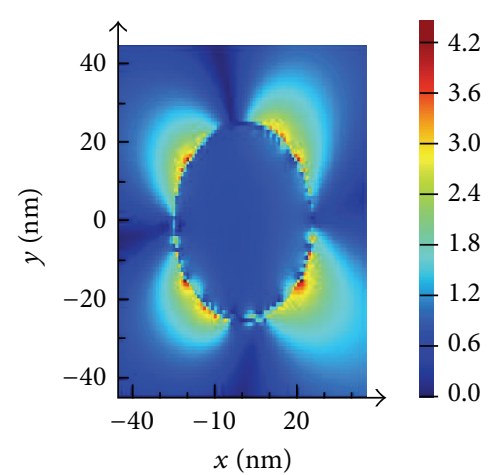

(b) 15 degrees

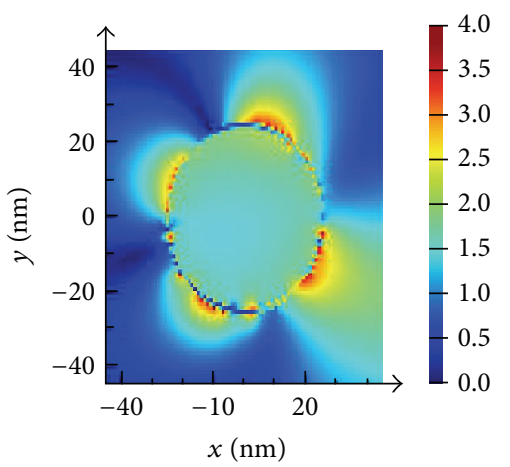

(e) 45 degrees- $380 \mathrm{~nm}$

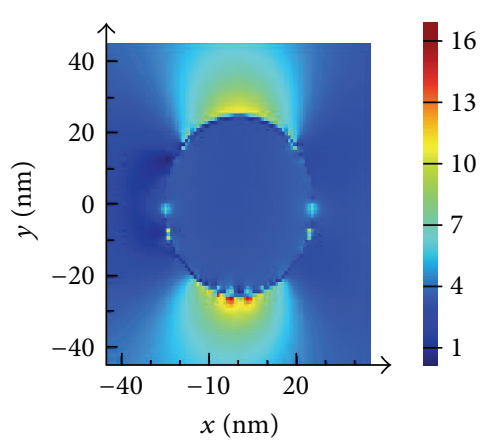

(h) 60 degrees $-479 \mathrm{~nm}$

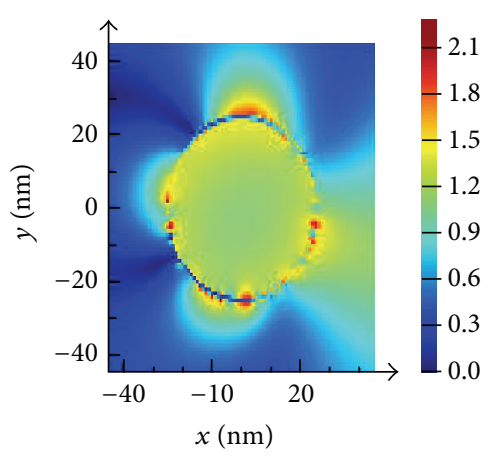

(k) 90 degrees- $380 \mathrm{~nm}$

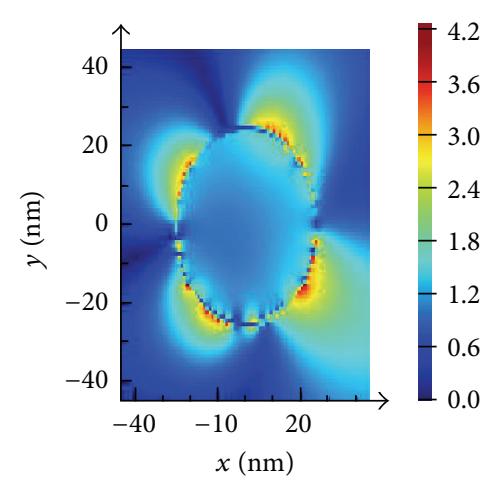

(c) 30 degrees $-380 \mathrm{~nm}$

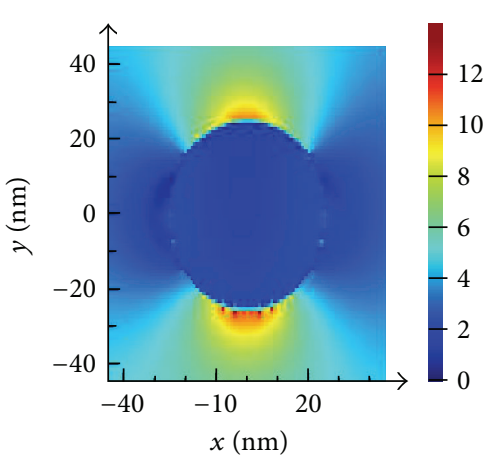

(f) 45 degrees- $591 \mathrm{~nm}$

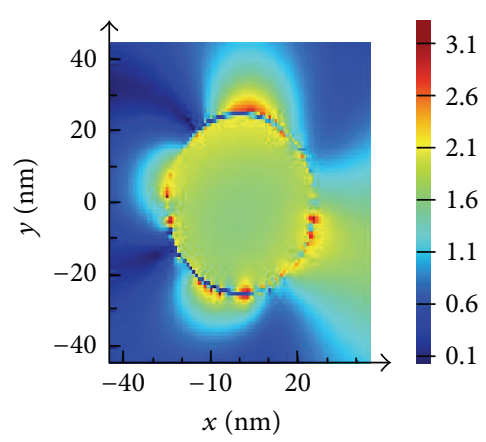

(i) 75 degrees $-380 \mathrm{~nm}$

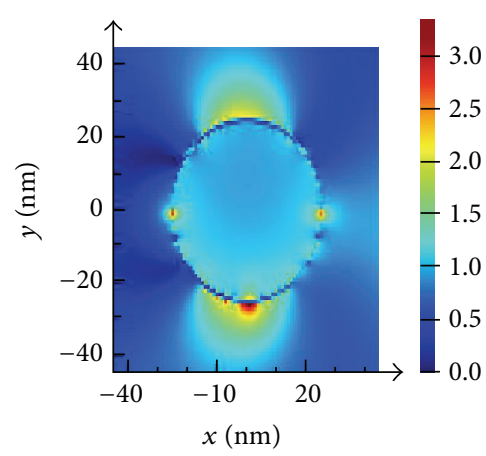

(l) 90 degrees $-416 \mathrm{~nm}$

FIGURE 5: Near-field distribution of silver nanoparticles. 


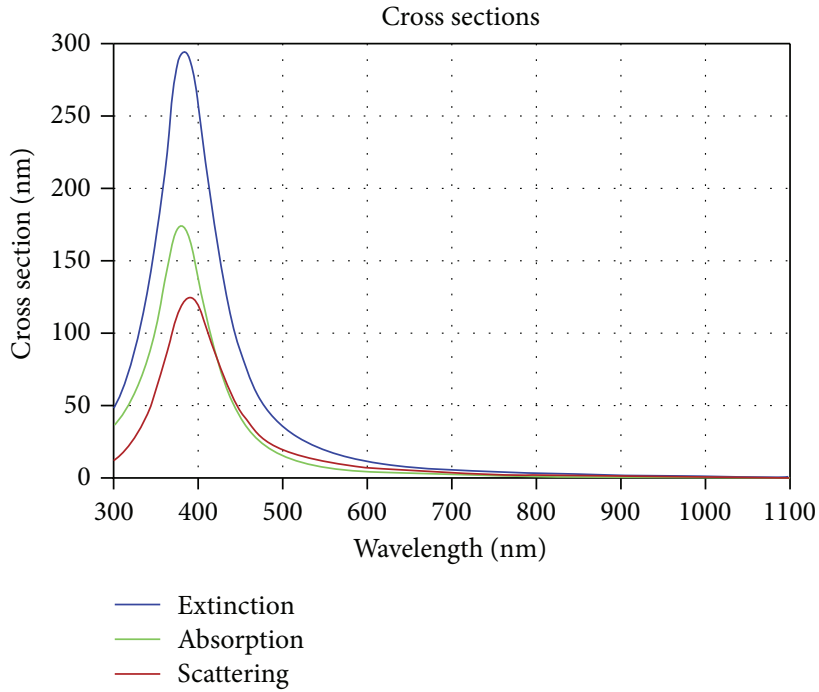

(a)

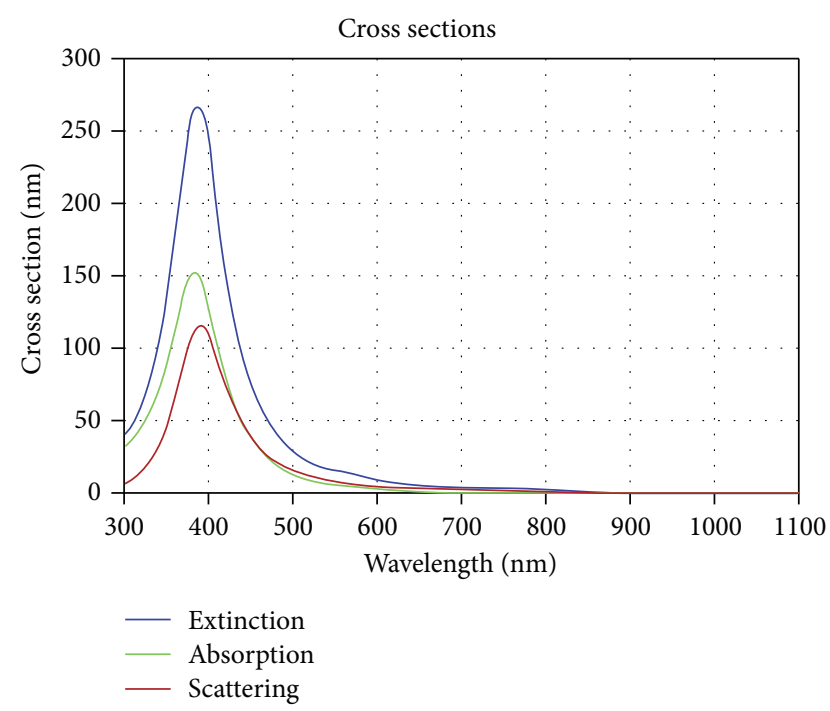

(b)

FIGURE 6: Extinction spectrum of different positions (far-field characteristics): (a) $20 \mathrm{~nm}$ downward deviation of the interface and (b) $20 \mathrm{~nm}$ upward deviation of the interface.

interface is studied. There are two resonance peaks and one of them appears to achieve a blue shift compared to the isotropic medium. LSPR effect within H-PDLC grating is the result of combined factors of LSPR of single nanoparticle and has a significant effect on the electric-optical properties of $\mathrm{H}$ PDLC grating. It will be a good theoretical guidance for the experiment research.

\section{Conflict of Interests}

The authors declare that there is no conflict of interests regarding the publication of this paper.

\section{Acknowledgments}

This research was supported by the National Key Foundation for Exploring Scientific Instrument of China (2012YQ15008720), the Key Research Project from Shanghai Education Committee (14ZZ138), Shanghai Key Subject Construction Funding (s30502), and the Development Fund for Shanghai Talents (2012026).

\section{References}

[1] C. Noguez, Plasmonics Fundamentals and Applications, Springer, New York, NY, USA, 2007.

[2] M. L. Brongersma and P. G. Kik, Surface Plasmon Nanophotonics, Springer, Amsterdam, The Netherlands, 2007.

[3] P. K. Jain, K. S. Lee, I. H. El-Sayed, and M. A. El-Sayed, "Calculated absorption and scattering properties of gold nanoparticles of different size, shape, and composition: applications in biological imaging and biomedicine," Journal of Physical Chemistry B, vol. 110, no. 14, pp. 7238-7248, 2006.

[4] K.-S. Lee and M. A. El-Sayed, "Gold and silver nanoparticles in sensing and imaging: sensitivity of plasmon response to size, shape, and metal composition," Journal of Physical Chemistry B, vol. 110, no. 39, pp. 19220-19225, 2006.

[5] A. S. Grimault, A. Vial, and M. L. de la Chapelle, "Modeling of regular gold nanostructures arrays for SERS applications using a 3D FDTD method," Applied Physics B: Lasers and Optics, vol. 84, no. 1-2, pp. 111-115, 2006.

[6] Z.-Y. Zhang and Y.-P. Zhao, "Extinction spectra and electrical field enhancement of Ag nanorods with different topologic shapes," Journal of Applied Physics, vol. 102, no. 11, Article ID 113308, 2007.

[7] T. J. Bunning, L. V. Natarajan, V. P. Tondiglia, and R. L. Sutherland, "Holographic polymer-dispersed liquid crystals (H-PDLCs)," Annual Review of Materials Science, vol. 30, no. 1, pp. 83-115, 2000.

[8] H. Yamane, H. Kikuchi, and T. Kajiyama, "Laser-addressing rewritable optical information storage of (liquid crystalline side chain copolymer/liquid crystals/photo-responsive molecule) ternary composite systems," Polymer, vol. 40, no. 17, pp. 47774785, 1999.

[9] U. Maschke, X. Coqueret, and M. Benmouna, "Electro-optical properties of polymer-dispersed liquid crystals," Macromolecular Rapid Communications, vol. 23, no. 3, pp. 159-170, 2002.

[10] J. Zou and J. Fang, "Adhesive polymer-dispersed liquid crystal films," Journal of Materials Chemistry, vol. 21, no. 25, pp. 91499153, 2011.

[11] H. S. Nalwa, Handbook of Advanced Electronic and Photonic Materials and Devices, Acadamic Press, San Diego, Calif, USA, 2001.

[12] F. P. Nicoletta, G. Chidichimo, D. Cupelli et al., "Electrochromic polymer-dispersed liquid-crystal film: a new bifunctional device," Advanced Functional Materials, vol. 15, no. 6, pp. 995999, 2005.

[13] J. Zhang, S. Yoshikado, and T. Aruga, "Shift multiplexing for holographic storage system using fiber bundle referencing," Applied Physics Letters, vol. 82, no. 1, pp. 25-27, 2003.

[14] Y. J. Liu, X. W. Sun, J. H. Liu, H. T. Dai, and K. S. Xu, "A polarization insensitive $2 \times 2$ optical switch fabricated by liquid 
crystal polymer composit," Applied Physics Letters, vol. 86, no. 4, pp. 1115-1117, 2005.

[15] M. S. Li, S. T. Wu, and A. Y.-G. Fuh, "Sensor for monitoring the vibration of a laser beam based on holographic polymer dispersed liquid crystal films," Optics Express, vol. 18, no. 25, pp. 26300-26306, 2010.

[16] S. Song, J. Jeong, S. H. Chung, S. M. Jeong, and B. Choi, "Electroluminescent devices with function of electro-optic shutter," Optics Express, vol. 20, no. 19, pp. 21074-21082, 2012.

[17] H. T. Dai, X. W. Sun, D. Luo, and Y. J. Liu, "Airy beams generated by a binary phase element made of polymer-dispersed liquid crystals," Optics Express, vol. 17, no. 22, pp. 19365-19370, 2009.

[18] G. Nabil, W. F. Ho, and H. P. Chan, "Experimental study on the performance of a variable optical attenuator using polymer dispersed liquid crystal," Applied Optics, vol. 52, no. 22, pp. E15E21, 2013.

[19] H. C. Lin, N. Collings, M. S. Chen, and Y. H. Lin, "A holographic projection system with an electrically tuning and continuously adjustable optical zoom," Optics Express, vol. 20, no. 25, pp. 27222-27229, 2012.

[20] G. M. Zharkova, A. P. Petrov, I. V. Samsonova, S. A. Strel'tsov, and V. M. Khachaturyan, "Polarization characteristics of polymer-liquid-crystal diffraction gratings," Journal of Optical Technology, vol. 75, no. 8, pp. 514-517, 2008.

[21] D. Cupelli, F. P. Nicoletta, G. D. Filpo et al., "Fine adjustment of conductivity in polymer-dispersed liquid crystals," Applied Physics Letters, vol. 85, no. 15, pp. 3292-3294, 2004.

[22] W. Ma, H. Yang, J. Liu, Z. Ni, D. Tang, and J. Yao, "Influences of shape on extinction spectrum for metal nanoparticles," Acta Optica Sinica, vol. 30, no. 9, pp. 2629-2633, 2010.

[23] J. Klosterman, L. V. Natarajan, V. P. Tondiglia et al., "The influence of surfactant in reflective HPDLC gratings," Polymer, vol. 45, no. 21, pp. 7213-7218, 2004.

[24] Y. J. Liu, X. W. Sun, H. T. Dai, J. H. Liu, and K. S. Xu, "Effect of surfactant on the electro-optical properties of holographic polymer dispersed liquid crystal Bragg gratings," Optical Materials, vol. 27, no. 8, pp. 1451-1455, 2005.

[25] J. Zheng, K. Gui, M. Zhang, K. Wang, C. Guo, and S. Zhuang, "The frequency modulation electro-optical response of holographic polymer dispersed liquid crystal display doped with nano Ag," Journal of Display Technology, vol. 10, no. 3, pp. 215222, 2014.

[26] Y. Liu, F. Song, J. Zhang et al., “Tunable local surface plasmon resonance in liquid-crystal-coated Ag nanoparticles," Physics Letters A, vol. 377, no. 16-17, pp. 1199-1204, 2013.

[27] Z. Yang, S. Chen, P. Fang, B. Ren, H. H. Girault, and Z. Tian, "LSPR properties of metal nanoparticles adsorbed at a liquidliquid interface," Physical Chemistry Chemical Physics, vol. 15, no. 15, pp. 5374-5378, 2013. 

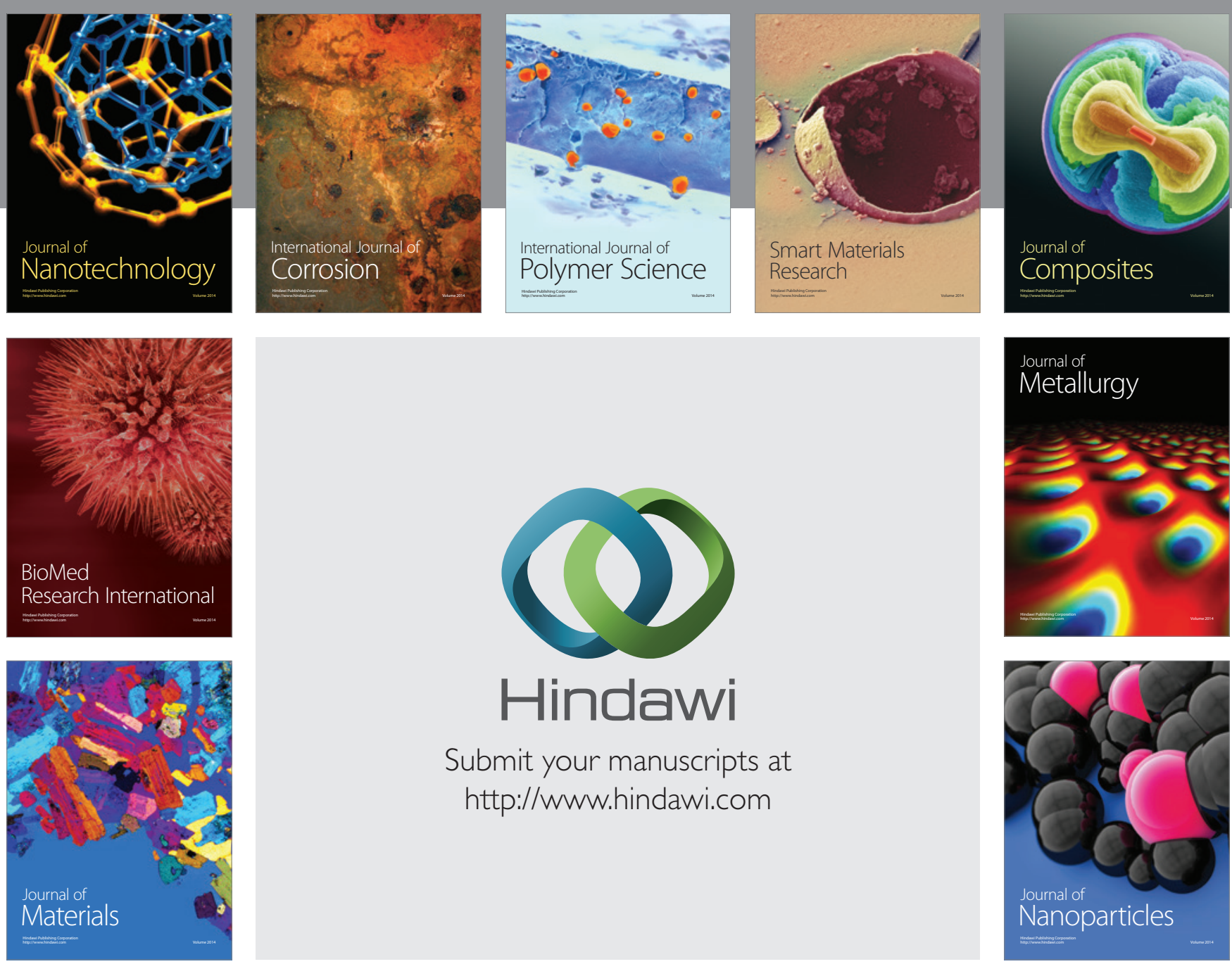

Submit your manuscripts at http://www.hindawi.com
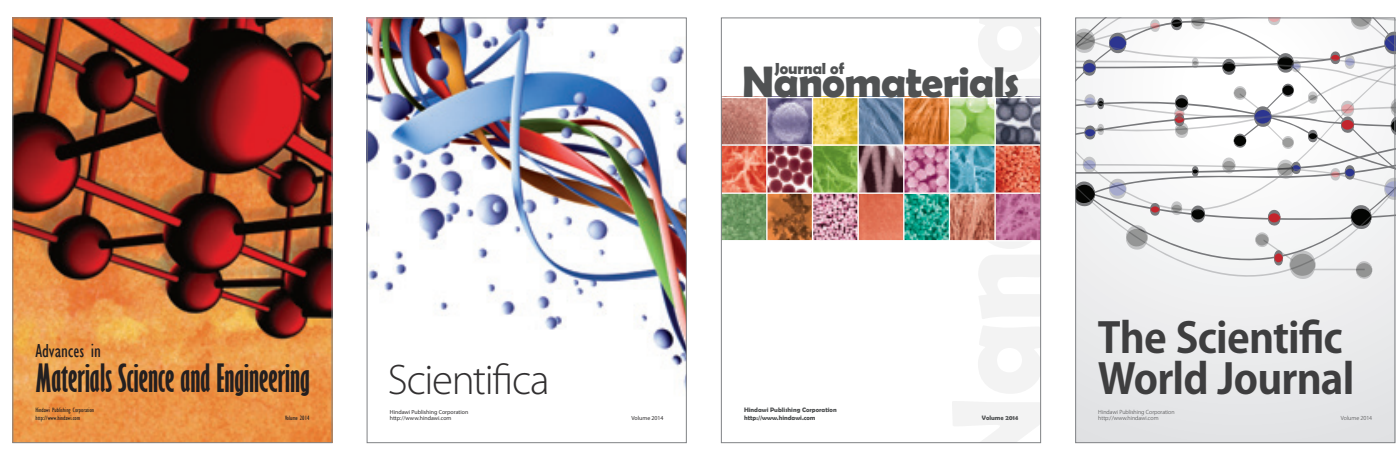

\section{The Scientific World Journal}
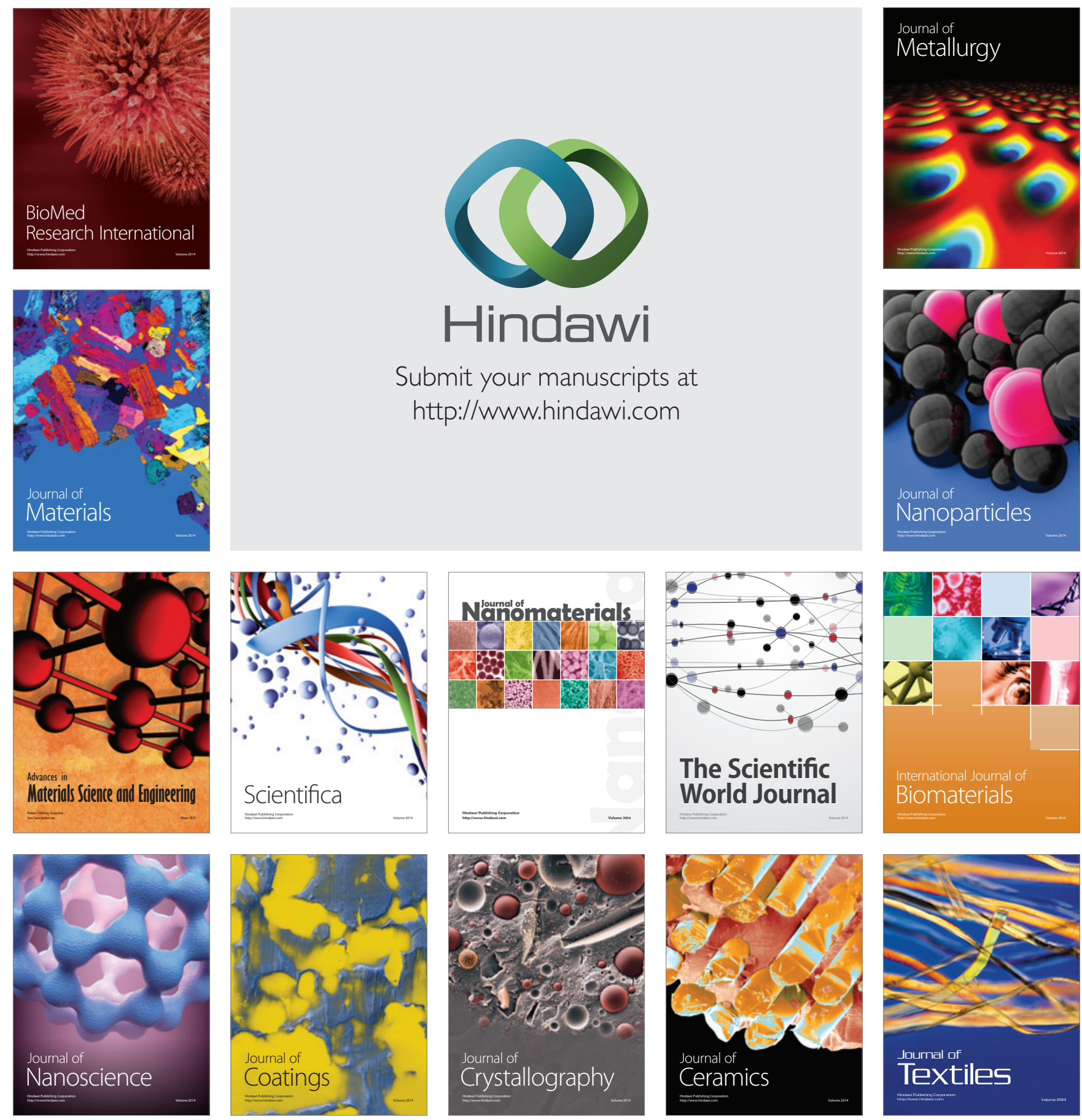